\title{
Universal Relaxation in a Holographic Metallic Density Wave Phase
}

\author{
Andrea Amoretti* \\ Dipartimento di Fisica, Università di Genova, via Dodecaneso 33, I-16146 Genova, Italy \\ and I.N.F.N.-Sezione di Genova and Physique Théorique et Mathématique and \\ International Solvay Institutes Université Libre de Bruxelles, C.P. 231, 1050 Brussels, Belgium \\ Daniel Areán ${ }^{\dagger}$ \\ Instituto de Física Teórica UAM/CSIC, Calle Nicolás Cabrera 13-15, Cantoblanco, 28049 Madrid, Spain \\ Blaise Goutéraux $\oplus^{\ddagger}$ \\ CPHT, CNRS, Ecole polytechnique, IP Paris, F-91128 Palaiseau, France and Nordita, \\ KTH Royal Institute of Technology and Stockholm University, \\ Roslagstullsbacken 23, SE-106 91 Stockholm, Sweden \\ Daniele Musso ${ }^{\S}$ \\ Departamento de Física de Partículas, Universidade de Santiago de Compostela \\ and Instituto Galego de Física de Altas Enerxías (IGFAE), Santiago de Compostela, 15705, Spain
}

(Received 5 July 2019; revised manuscript received 4 September 2019; published 19 November 2019)

In this Letter, we uncover a universal relaxation mechanism of pinned density waves, combining gaugegravity duality and effective field theory techniques. Upon breaking translations spontaneously, new gapless collective modes emerge, the Nambu-Goldstone bosons of broken translations. When translations are also weakly broken (e.g., by disorder or lattice effects), these phonons are pinned with a mass $m$ and damped at a rate $\Omega$, which we explicitly compute. This contribution to $\Omega$ is distinct from that of topological defects. We show that $\Omega \simeq G m^{2} \Xi$, where $G$ is the shear modulus and $\Xi$ is related to a diffusivity of the purely spontaneous state. This result follows from the smallness of the bulk and shear moduli, as would be the case in a phase with fluctuating translational order. At low temperatures, the collective modes relax quickly into the heat current, so that late time transport is dominated by the thermal diffusivity. In this regime, the resistivity in our model is linear in temperature and the ac conductivity displays a significant rearranging of the degrees of freedom, as spectral weight is shifted from an off-axis, pinning peak to a Drude-like peak. These results could shed light on transport properties in cuprate high $T_{c}$ superconductors, where quantum critical behavior and translational order occur over large parts of the phase diagram and transport shows qualitatively similar features.

DOI: 10.1103/PhysRevLett.123.211602

The spontaneous formation of charge density waves and other types of translational order in weakly coupled onedimensional metals is well understood: the Peierls instability opens a gap and a collective mode of electron-hole pairs is formed [1]. After condensation, the spectrum of the system contains a gapless mode (the phase of the condensate, ie the Goldstone boson generated by the spontaneous breaking of translations): physically, the density wave can slide without energy cost. In the presence of weak disorder or lattice effects, the Goldstone (or with a slight

Published by the American Physical Society under the terms of the Creative Commons Attribution 4.0 International license. Further distribution of this work must maintain attribution to the author(s) and the published article's title, journal citation, and DOI. Funded by SCOAP ${ }^{3}$. abuse of terminology, phonon) is pinned. More formally, weak explicit breaking of translations generates a small phonon mass $m$.

Pinning has dramatic consequences on the linear frequency-dependent response. Assuming a normal metallic state with an ac conductivity characterized by a Drude-like peak centered at zero frequency, the spatially modulated state is characterized by a transfer of spectral weight to finite frequencies: an off-axis peak is formed at the socalled pinning frequency $\omega_{o} \sim m$. In classic treatments [1], the Goldstone contribution vanishes at zero frequency and the system is an electrical insulator.

The pinning peak is also characterized by its width, which heuristically captures the typical lifetime of excitations of the system. There are two well-known contributions to the peak width [1]. One comes from the rate $\Gamma$ at which the momentum of the system relaxes due to disorder 
or lattice effects. The other comes from the rate $\Omega$ at which the Goldstone relaxes in the presence of mobile defects [2]. In direct analogy to how vortices relax superfluid phase gradients and eventually destroy superfluidity, dislocations and disclinations also spoil phase coherence of the density wave. The phonon contributes to the dc conductivity $\sigma_{\mathrm{dc}} \simeq \omega_{p}^{2} \Omega /\left(\Gamma \Omega+\omega_{o}^{2}\right)$ where $\omega_{p}$ is the plasma frequency (the Drude weight), [3]. As temperature decreases, defects freeze out, leading to vanishing phase relaxation and insulating behavior.

Density waves play an important role in the phase diagram of cuprate high $T_{c}$ superconductors. These materials are doped Mott insulators [4], which tend to order translationally upon doping [5-12]. In the strange metallic phase, their resistivity scales linearly with temperature [13] and violates the Mott-Ioffe-Regel limit [14]. This signals the breakdown of the quasiparticle picture and is usually interpreted as a sign of strongly coupled dynamics. This behavior has been attributed to an underlying metallic quantum critical point with maximal, Planckian dissipation $[15,16]$ characteristic of strongly coupled phases.

In the strange metallic phase, a shift of spectral weight is often observed with sharp Drude peaks at low temperatures moving off axis as temperature increases [17]. Quantum melting of stripe order by proliferating defects has been proposed as the origin of the strange metallic phase-see, e.g., $[7,18]$ — but microscopic descriptions of fluctuating stripes in the vicinity of a quantum critical metallic phase and their effects on the ac conductivity remain a challenge [19-22]. Based on an effective field theory approach (hydrodynamics), [23] argued that fluctuating translational order could cause the shift in spectral weight mentioned above. However the relaxation parameters $\Omega, \Gamma$, and $\omega_{o}$ enter as an input in the hydrodynamic theory, and need to be computed by a microscopic theory valid at strong coupling.

In this Letter, we combine gauge-gravity duality with hydrodynamics [3] to study the long distance transport properties of strongly coupled, weakly pinned density waves in the vicinity of a metallic quantum critical phase with a $T$-linear resistivity. Gauge-gravity duality allows us to address the strongly coupled dynamics of certain quantum field theories by mapping them to a weakly coupled theory of gravity [24-27].

We identify unambiguously a new contribution to the phase relaxation rate $\Omega$ due to weak explicit breaking of translations, which drives the shift in spectral weight and dominates the resistivity. While this type of damping is well known from studies of the magnetic field-induced melting of Wigner solids [28,29], and phonon damping by disorder was studied very early on-see, e.g., [30,31]—it was reported to affect the ac conductivity only through the pinning frequency $\omega_{o}$ and the momentum relaxation rate $\Gamma[1,3]$. Our results should also shed light on previous holographic studies of pinned density waves [32-38], where this contribution was not explicitly identified. In passing, we verify the validity of the hydrodynamic theory of damped and pinned density waves written in [3].

Furthermore, we uncover a universal relation between the phonon damping rate and its mass,

$$
\Omega \simeq G m^{2} \Xi=\chi_{\pi \pi} \omega_{o}^{2} \Xi \Rightarrow \rho_{\mathrm{dc}} \equiv \sigma_{d c}^{-1} \simeq \frac{1}{\rho^{2} \Xi} .
$$

$\Xi$ is a phonon diffusive transport coefficient, $G$ the phonon shear modulus, $\rho$ the charge density, $\chi_{\pi \pi}$ the momentum static susceptibility, and the pinning frequency is defined from the phonon mass as $\omega_{o}^{2} \equiv \mathrm{Gm}^{2} / \chi_{\pi \pi}$. It is universal since it determines microscopic parameters in terms of universal thermodynamic and hydrodynamic data. The expression for the resistivity is reminiscent of an Einstein relation. We show that this relation is true in the limit of small bulk and shear moduli. We expect it will hold more generally in phases with fluctuating translational order, or close to a phase transition towards a translationally ordered phase, where these quantities are small.

Remarkably, at low temperatures, we observe that the system saturates a bound ensuring the positivity of entropy production. This is naturally explained by relaxation of the phonons into the heat current. The resistivity is then controlled by the thermal diffusivity $D_{T} \sim 1 / T$. Our result resonates with the idea that the thermalization time in strange metals and other strongly coupled quantum phases is bounded from below by the "Planckian" timescale $\tau_{P} \sim \hbar /\left(k_{B} T\right)[15,16]$ and that production of entropy is minimal, e.g., through a lower bound on the shear viscosity [39-41].

We now explain in more detail how we arrive at these results, and close by commenting further on the relevance of our results to strange metals.

Holographic model.-We consider the holographic model [42] $S=\int d^{4} x \sqrt{-g} \mathcal{L}$

$$
\mathcal{L}=R-\frac{1}{2} \partial \phi^{2}-V(\phi)-\frac{1}{4} Z(\phi) F^{2}-\frac{1}{2} \sum_{I=1}^{2} Y(\phi) \partial \psi_{I}^{2},
$$

with the scalar couplings behaving near the anti-de Sitter (AdS) boundary as $V_{u v}(\phi)=-6-\phi^{2}+O\left(\phi^{3}\right)$, $Z_{u v}(\phi)=1+O(\phi), Y_{u v}(\phi)=\phi^{2}+O\left(\phi^{3}\right)$. For concreteness, in our numerical calculations we work with $V(\phi)=-6 \cosh (\phi / \sqrt{3}), Z(\phi)=\exp (-\phi / \sqrt{3})$, and $Y(\phi)=$ $(1-\exp \phi)^{2}$.

The model (2) enjoys a global shift symmetry $\psi_{I} \mapsto$ $\psi_{I}+c_{I}$. Adopting the background ansatz $\psi_{I}=k x^{I}$, $x^{I}=\{x, y\}$, the product of spacetime translations and shifts is broken to a diagonal $U(1)$. The $\psi_{I}$ then transform nonlinearly under translations precisely as Goldstones of broken translations are expected to. The other background 
fields can consistently be taken to depend solely on the holographic radial coordinate, $d s^{2}=-D(r) d t^{2}+B(r) d r^{2}+$ $C(r)\left(d x^{2}+d y^{2}\right), \phi=\phi(r)$, and $A=A(r) d t$. Near the AdS boundary $r \rightarrow 0, \phi(r)=\lambda r+\phi_{(v)} r^{2}+O\left(r^{3}\right)$, where $\lambda$ is the source and $\phi_{(v)}$ the vev of the operator dual to $\phi$. Moreover, the bulk scalar fields can be rewritten as complex scalars $\Phi_{I}=\phi \exp \left(i \psi_{I}\right)$ close to the boundary. This shows that the breaking is spontaneous when $\lambda=0$ or explicit when $\lambda \neq 0$ [43-45], which is the main focus of this Letter.

This holographic model does not capture the phase transition between the normal and the ordered phase. Instead, it describes the low energy dynamics of the (pseudo)phonons in the ordered phase, consistent with the pattern of symmetry breaking in an isotropic, twodimensional Wigner crystal (WC) [46].

Finite temperature, finite density states are modeled by charged black holes in the bulk, which implies the existence of a regular Killing horizon at $r=r_{h}$. Hereafter, we use a subscript $h$ to denote quantities evaluated at $r=r_{h}$.

Unrelaxed WC hydrodynamics from holography.Aspects of unrelaxed WC hydrodynamics were studied previously in $[44,45]$, and are reviewed in the Supplemental Material [50]. The hydrodynamic retarded Green's functions at zero wave vector for the electric current and the phonons are [3,51]

$$
\begin{aligned}
G_{j j}^{R} & =\frac{\rho^{2}}{\chi_{\pi \pi}}-i \omega \sigma_{o}, \quad G_{j \varphi}^{R}=-\gamma_{1}-\frac{\rho}{\chi_{\pi \pi}} \frac{i}{\omega}, \\
G_{\varphi \varphi}^{R} & =\frac{1}{\chi_{\pi \pi} \omega^{2}}-\Xi \frac{i}{\omega} .
\end{aligned}
$$

$\sigma_{o}, \gamma_{1}$, and $\Xi$ are diffusive transport coefficients that appear in the longitudinal sector of hydrodynamic constitutive relations at first order in gradients [3,50]. $\chi_{\pi \pi}=s T+\mu \rho+k^{2} I_{Y}$, with the entropy density $s$ given by the horizon area, $\rho$ by the electric flux out of the horizon, and the chemical potential $\mu=A(0)$. The bulk and shear moduli are $2 K=G=k^{2} I_{Y}+O\left(k^{4}\right)=k^{2} \int_{0}^{r_{h}} \sqrt{B D} Y+$ $O\left(k^{4}\right)$ [44,48]. $\sigma_{o}$ has been computed in [44,45,64], and the computation of $\gamma_{1}$ and $\Xi$ is presented in the Ref. [52]:

$$
\begin{aligned}
\sigma_{o} & =\frac{(s T+\mu \rho)^{2}}{\left(\chi_{\pi \pi}\right)^{2}} Z_{h}+\frac{4 \pi \rho^{2} k^{2}\left(I_{Y}\right)^{2}}{s Y_{h}\left(\chi_{\pi \pi}\right)^{2}}, \\
\gamma_{1} & =-\frac{4 \pi I_{Y} \rho(s T+\mu \rho)}{s Y_{h}\left(\chi_{\pi \pi}\right)^{2}}-\mu \frac{\left(s T+k^{2} I_{Y}\right)}{\left(\chi_{\pi \pi}\right)^{2}} Z_{h}, \\
\Xi & =\frac{4 \pi(s T+\mu \rho)^{2}}{k^{2} s Y_{h}\left(\chi_{\pi \pi}\right)^{2}}+\frac{\mu^{2} Z_{h}}{\left(\chi_{\pi \pi}\right)^{2}} .
\end{aligned}
$$

We have verified that these expressions match the correlators computed numerically and that the phonons are dual to the vevs of the bulk fields $\psi_{I}$ [52]. After turning on

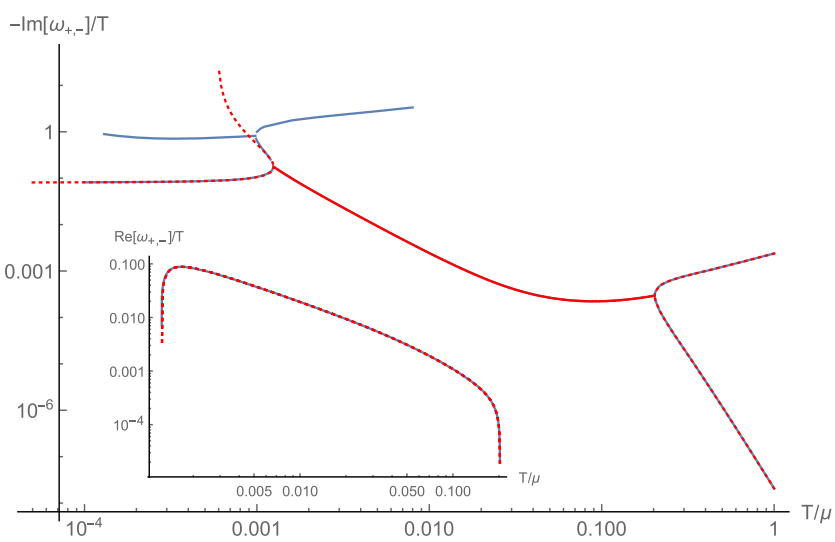

FIG. 1. $-\operatorname{Im}\left[\omega_{ \pm}\right]$vs temperature. No visible difference between the exact numerics (solid, blue) and the analytical approximation using (6) and (8) (dashed red). Inset: real part.

relaxation, these coefficients only receive small $O(\lambda)$ corrections, which can be safely neglected.

Relaxed WC hydrodynamics from holography.-To describe weak explicit breaking of translations, we assume $\lambda / \mu \ll \phi_{(v)} / \mu^{2}$. We also take $\lambda / \mu \ll k / \mu \ll 1$. In our numerics, we choose $k / \mu=0.1$ and $\lambda / \mu=-10^{-5}$. At zero wave vector $q$, WC hydrodynamics predicts for the ac conductivity $[3,50]$

$$
\sigma(\omega)=\sigma_{o}+\frac{\omega_{o}^{2} \gamma_{1}\left(2 \rho-i \gamma_{1} \chi_{\pi \pi} \omega\right)-\frac{\rho^{2}}{\chi_{\pi \pi}}(\Omega-i \omega)}{\omega^{2}-\omega_{o}^{2}+i \omega \Omega} .
$$

We have set $\Gamma=0$, consistent with our numerics (see [50]). There are two gapped poles, which capture the relaxation of the phonons and of momentum:

$$
\omega_{ \pm}=-\frac{i}{2} \Omega \pm \frac{1}{2} \sqrt{4 \omega_{o}^{2}-\Omega^{2}} .
$$

In Fig. 1, we compute numerically the lightest pair of quasinormal modes (QNMs) of the holographic system at zero wave vector [50] and match their location to (6), see Fig. 2.

We also find that $\Omega \sim|\lambda|, \omega_{o} \sim|\lambda|^{1 / 2} k$ [50] and $G, K \sim k^{2}$, so that $m \sim|\lambda|^{1 / 2}$ [65]. This implies that both $\Omega$ and $m$ can be extracted in the limit $k=0$, i.e., small bulk and shear modulus. In this limit, the hydrodynamic correlator of the transverse phonon is

$$
G_{\varphi \varphi}^{R}(\omega, q)=\frac{\Omega+q^{2} G \Xi}{G\left(q^{2}+m^{2}\right)\left(i \omega-\Omega-q^{2} G \Xi\right)} .
$$

Near the boundary, $\delta \psi_{x}(r)=\delta \psi_{x,(0)}+\delta \psi_{x,(1)} r+O\left(r^{2}\right)$, where $\delta \psi_{x,(0)}$ is the source and $\left\langle O_{\delta \psi}\right\rangle=\lambda^{2} \delta \psi_{x,(1)}$ the vev. In [50], we show that the phonon is $G^{2} \varphi=k\left\langle O_{\delta \psi}\right\rangle$. The pseudodiffusive pole at $\omega \simeq-i \Omega-i G \Xi q^{2}$ is a consequence 


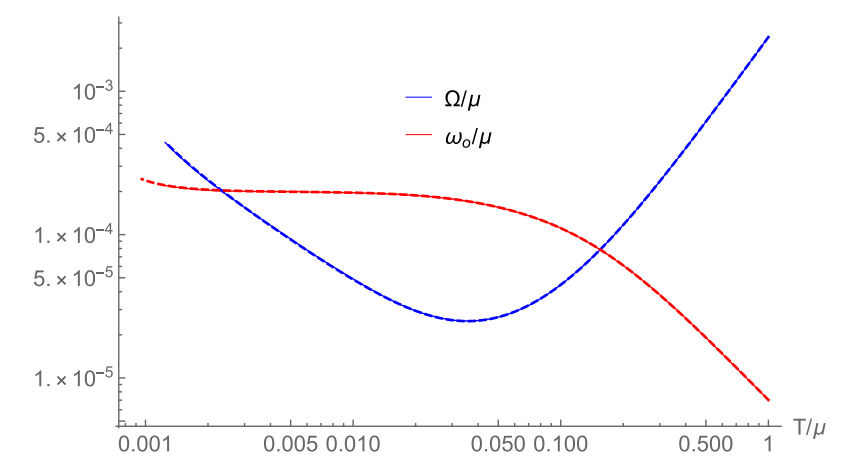

FIG. 2. $\Omega / \mu$ (blue) and $\omega_{o} / \mu$ (red) vs $T / \mu$. No visible difference between the exact numerical result from the QNMs location (solid line) and the analytical approximation (8) (dashed), evaluated on the $k \neq 0, \lambda \neq 0$ numerical background.

of the explicit breaking of the global shift symmetry when $\lambda \neq 0$. We also compute:

$$
\Omega \simeq \frac{I_{Y} m^{2}}{C_{h} Y_{h}} \simeq \frac{1}{\int_{0}^{r_{h}} d r\left(\frac{C_{h} Y_{h} \sqrt{B}}{C Y \sqrt{D}}-\frac{1}{4 \pi T} \frac{1}{r_{h}-r}\right)} .
$$

The match to the exact numerics is excellent, see Figs. 1 and 2 .

At small $k / \mu$, (4) becomes $\Xi \simeq 1 /\left(k^{2} C_{h} Y_{h}\right)+O\left(k^{0}\right)$. Putting this together with (8) leads to the relation (1) above. This is one of our key results, and shows that the relaxation of the phonon is entirely governed by a diffusivity of the unrelaxed theory in the entire range of temperatures where WC hydrodynamics applies.

Assuming $\lambda \neq 0$, the holographic Ward identity governing momentum relaxation is $\partial_{t} \pi^{i} \equiv \dot{\pi}^{i}=k \delta \psi_{i,(1)}$, which shows that $k$ controls the strength of momentum relaxation. Recall that $\omega_{o}^{2} \sim k^{2}$ while $\Omega \sim k^{0}$. Restoring $\Gamma$ in the WC hydrodynamic expressions for the retarded Green's functions,

$$
\begin{gathered}
\Gamma+\frac{\omega_{o}^{2}}{\Omega}=\frac{1}{\chi_{\pi \pi}} \lim _{\omega \rightarrow 0} \lim _{k \rightarrow 0} \frac{1}{\omega} \operatorname{Im} G_{\tilde{\pi}^{x} \pi^{x}}^{R}(\omega, q=0), \\
\frac{\omega_{o}^{2}}{\Omega^{2}}=-\frac{1}{\chi_{\pi \pi}} \lim _{\omega \rightarrow 0} \lim _{k \rightarrow 0} \operatorname{Re} G_{\pi^{x} \pi^{x}}^{R}(\omega, q=0) .
\end{gathered}
$$

The Ward identity relates both quantities on the left-hand side to $G_{\psi_{x} \psi_{x}}^{R}$ evaluated at $k=0$ [50], leading to the same expressions as in (8).

Charge transport at high temperature.-As in many conventional systems where translations are broken spontaneously [1], our holographic system is an electrical insulator $\left(d \rho_{\mathrm{dc}} / d T<0\right)$ at high $T \gtrsim T_{\mathrm{qc}} \simeq 5.10^{-2} \mu$.

Its de conductivity is $\sigma_{\mathrm{dc}}=Z_{h}+\rho^{2} /\left(k^{2} C_{h} Y_{h}\right)$, [53,67]. Our numerics reveal that the dc conductivity is dominated by phase relaxation, $\sigma_{\mathrm{dc}} \equiv \sigma(0) \simeq \rho^{2} \Omega /\left(m^{2} G\right)$.

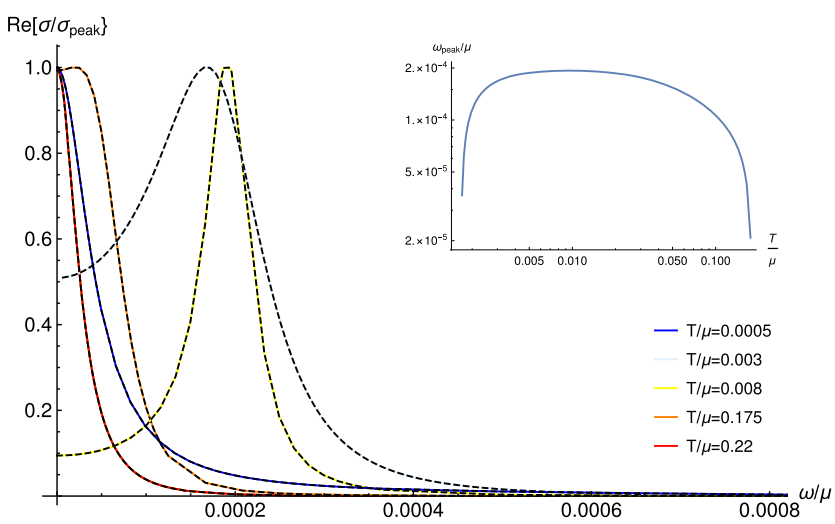

FIG. 3. No visible difference between $\operatorname{Re}[\sigma]$ (normalized with respect to its maximal value $\sigma_{\text {peak }}$ ) computed numerically and hydrodynamic predictions (5) and (11) (black dashed lines). The ac conductivity above $T_{\mathrm{cm}} / \mu \simeq 10^{-3}$ shows an off-axis peak, which moves back on axis as $T \lesssim T_{\mathrm{cm}}$. Inset: temperature dependence of the peak location.

As $T$ decreases, the ac conductivity (see Fig. 3) shows a pinning peak moving away from zero frequency, $d \omega_{\text {peak }} / d T<0$. Correspondingly, the two poles $\omega_{ \pm}$collide, acquire a real gap and move away from the imaginary axis at low temperatures, see Fig. 1. The match between the line shape of the ac conductivity and the hydrodynamic prediction (5) is excellent. $\Omega$ and $\omega_{o}$ are obtained from the location of the QNMs. We emphasize that there is no further fitting: $\sigma_{o}$ and $\gamma_{1}$ are computed using (4), $\rho$ and $\chi_{\pi \pi}$ directly from the $\lambda \neq 0$ numerical background.

Charge transport at low temperatures.-For temperatures $T \lesssim T_{\mathrm{qc}} \simeq 5.10^{-2} \mu$, the bulk geometry undergoes a qualitative change and becomes conformal to $\mathrm{AdS}_{2} \times \mathrm{R}^{2}$ near the horizon $[44,45]$, with $s \sim T$ and metallic behavior $\rho_{\mathrm{dc}} \sim T$-see, e.g., [40,50,54].

As the temperature is lowered, the two off-axis QNMs turn around and move back towards the imaginary axis, see Fig. 1.

Accordingly, the off-axis peak in $\operatorname{Re} \sigma(\omega)$ reverses direction, $d \omega_{\text {peak }} / d T>0$, and moves back towards zero frequency, see Fig. 3. In this region, small deviations between the hydrodynamic formula (5) and the exact ac conductivity computed numerically are observed, together with a small mismatch between the dc values of at most $\sim 1 \%$. The first reason is that a third QNM is coming closer. The second reason are small but finite departures $O\left[\omega_{o}^{2} /(\Omega T) \sim k^{2}\right]$ from the hydrodynamic formula (5) at low temperatures, similar to those reported in [68]. In contrast, at high $T$ these corrections $O\left[\omega_{o}^{2} /(\Omega T)\right]$ decay like a negative power of $T$, similar to [69].

Eventually, the two QNMs collide, and one of them moves back up the imaginary axis, dominating the dynamics at very low temperatures. The other QNM goes down the imaginary axis and collides at $T_{\mathrm{cm}} \simeq 10^{-3} \mu$ with the third QNM mentioned above, which signals the breakdown 
of WC hydrodynamics. $T_{\mathrm{cm}}$ is controlled by the magnitude of the explicit breaking scale $\lambda$ [50]. Below $T_{\mathrm{cm}}$, the system behaves like a fluid with slow momentum relaxation with a Drude-like conductivity [27]

$$
\sigma(\omega) \simeq \frac{\rho^{2}}{\chi_{\pi \pi}} \frac{1}{\Gamma-i \omega} \Rightarrow \rho^{\mathrm{dc}} \simeq \frac{\chi_{\pi \pi}}{\rho^{2}} \Gamma,
$$

see Fig. 3. The longest-lived QNM captures slowly relaxing momentum and controls the width of the Drude peak. Its location is predicted to be $\omega \simeq-i \Gamma=-i k^{2} C_{h} Y_{h} / \chi_{\pi \pi}$ [setting $\omega_{o}=0$ in the right hand side of (9) for purely explicit breaking]. In this regime, the phonons have decoupled from momentum: the two subdominant QNMs are very well approximated by the two longestlived QNMs of the $k=0 \delta \psi$ correlator (the phonons), and contribute negligibly to $\operatorname{Re} \sigma(\omega)$.

To summarize, we can distinguish between a high temperature phase with a crossover from insulating to metallic behavior as $T \lesssim T_{\mathrm{qc}}$, dominated by damped, pinned phonons as predicted by WC hydrodynamics [3]; and a low temperature metallic phase at $T \lesssim T_{\mathrm{cm}}$, where the charge dynamics of the system is simply governed by slow momentum relaxation, and the phonons are decoupled.

Universal low temperature relaxation.-Another universal aspect of relaxation in our model stems from positivity of entropy production in WC hydrodynamics, which places a bound on transport coefficients [50]

$$
\gamma_{1}^{2} \leq \min \left(\sigma_{o} \Xi, \frac{\Omega \sigma_{o}}{\chi_{\pi \pi} \omega_{o}^{2}}\right) .
$$

Due to (1), both terms on the right-hand side are equal. At temperatures $T \lesssim T_{\mathrm{qc}}$ marking the onset of the quantum critical phase, we observe from (4) (neglecting eg $s T$ terms vs $\mu \rho$ or $k^{2} I_{Y}$ ) and our numerics (see Fig. 4) that

$$
\gamma_{1} \simeq-\frac{\mu}{\chi_{\pi j_{q}}} \sigma_{o}, \quad \Xi \simeq \frac{\Omega}{m^{2} G} \simeq\left(\frac{\mu}{\chi_{\pi j_{q}}}\right)^{2} \sigma_{o}
$$

where $\chi_{\pi j_{q}}=\chi_{\pi \pi}-\mu \rho$ since $j_{q}=\pi-\mu j$ (from relativistic symmetry) and $\chi_{j \pi}=\rho$. The expressions (13) saturate the bound (12).

These results can be explained by considering the relaxation of the phonons into the heat current $j_{q}$, which is a universal mechanism in finite temperature systems. In particular, as translations are explicitly broken in our system, the only collective modes at the longest distances are diffusion of charge and energy. From WC hydrodynamics [3],

$$
\Xi=\lim _{\omega \rightarrow 0, q \rightarrow 0, \lambda \rightarrow 0} \frac{\operatorname{Im} G_{\dot{\varphi} \dot{\varphi}}^{R}(\omega, q)}{\omega},
$$

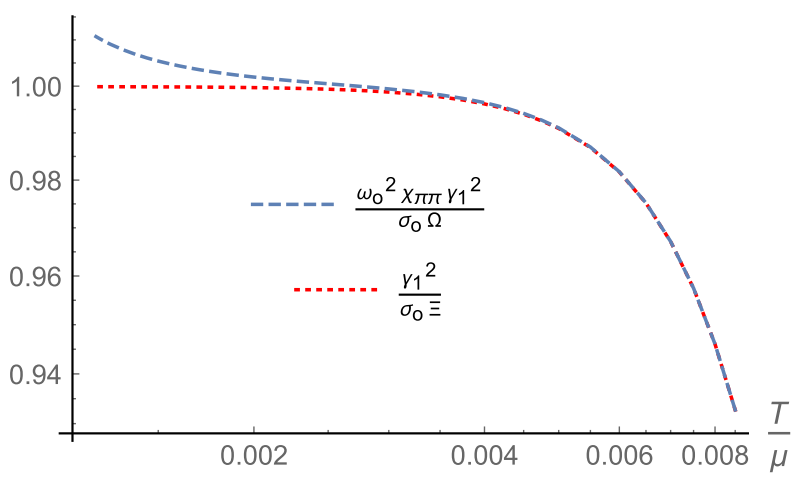

FIG. 4. The entropy bound of (un)relaxed WC hydrodynamics saturates as $T<T_{\mathrm{qc}}$. As $T \rightarrow T_{\mathrm{cm}}$, the bound of relaxed WC hydrodynamics starts to be violated.

$$
\frac{\Omega}{\omega_{o}^{2}}=\chi_{\pi \pi} \lim _{\omega \rightarrow 0, \lambda \rightarrow 0} \frac{\operatorname{Im} G_{\dot{\varphi} \dot{\varphi}}^{R}(\omega, q=0)}{\omega} .
$$

The limits $q \rightarrow 0$ and zero relaxation $\lambda \rightarrow 0$ do not commute. The $\omega \rightarrow 0$ limit must be taken last. The universal contribution to the Hamiltonian $\Delta \mathcal{H}_{q}=\int d^{2} x\left(\pi \cdot j_{q}\right) /$ $\left(\chi_{\pi j_{q}}\right)$ gives $\dot{\varphi}=i\left[\Delta \mathcal{H}_{q}, \varphi\right]=j_{q} / \chi_{\pi j_{q}}$. This leads to (13) using (14) and (15): in this regime relaxation is controlled by thermal, incoherent diffusion processes [70-72].

The small violation of the bound (12) as $T \rightarrow T_{\mathrm{cm}}$ signals the breakdown of WC hydrodynamics. As $\lambda \rightarrow 0$, $T_{\mathrm{cm}} \rightarrow 0$ as well, so that in the spontaneous limit WC hydrodynamics applies to arbitrarily low temperatures and the bound $\gamma_{1}^{2} \leq \sigma_{o} \Xi$ saturates without violation [52]. In [52], we also discuss how this depends on the scaling properties of the IR critical phase.

Outlook.-Experimental data in bad metals suggest $\omega_{\text {peak }} \sim k_{B} T / \hbar,[23]$ (see also $[73,74]$ for more recent experimental observations). In our holographic model, while $d \omega_{\text {peak }} / d T>0$, we do not observe $\omega_{\text {peak }} \sim k_{B} T / \hbar$. This is because in the region where $d \omega_{\text {peak }} / d T>0$, the phonon mass has a very weak dependence on temperature, while $\Omega \sim 1 / T$, consistent with the resistivity $\rho_{\mathrm{dc}} \simeq m^{2} / \Omega \sim T$.

A linear temperature dependence $\omega_{\text {peak }} \sim T$ would obtain if $m \sim \Omega \sim T$ [23], as expected for quantum fluctuations of an order parameter in the vicinity of a quantum critical point [15], provided $G \Xi \sim 1 / T$. For small bulk and shear modulus, this is indeed the behavior predicted by WC hydrodynamics in our system for the thermal diffusivity, $D_{T} \simeq \rho^{2} \Omega /\left(G m^{2}\right)+O\left(G^{0}, K^{0}\right) \sim 1 / T$. In strongly coupled systems, diffusivities are expected to saturate a lower bound $G \Xi \gtrsim \hbar v^{2} / k_{B} T$ [75], where $v$ is some characteristic velocity. This is the behavior reported in recent experiments on cuprates and cold atoms [76-78]. It would be interesting to further investigate if holographic models of pinned density waves can capture this behavior. 
Two distinct universal relaxation mechanisms are at play in our model. First, the smallness of the shear and bulk moduli implies $\Omega \simeq G m^{2} \Xi$ in the whole temperature regime where WC hydrodynamics applies $T \gtrsim T_{\mathrm{cm}}$. Independently, at low temperatures $T<T_{\mathrm{qc}}$, the phonons relax into the heat current leading to (13) (see also [79]). Universal relaxation by hydrodynamic operators was discussed previously in $[40,80]$ (see also [41]). This motivates a better characterization of the parameter space where these relaxation mechanisms dominate, including in other holographic models of pinned translational order [34-38,81], in order to consider further their applicability to real materials.

We would like to thank Riccardo Argurio, Marco Fazzi, Saso Grozdanov, Javier Mas, Alfonso Ramallo, and Javier Tarrío for stimulating and insightful discussions. We thank Richard Davison for comments on a previous version of the manuscript. We would also like to thank Tomas Andrade and Alexander Krikun for sharing with us an advanced draft of their work [38], which has some overlap with our results. B. G. would especially like to thank Luca Delacrétaz, Sean Hartnoll, and Anna Karlsson for numerous insightful discussions on Wigner crystal hydrodynamics and the various relaxation mechanisms for the phonons. B. G. has been partially supported during this work by the Marie Curie International Outgoing Fellowship No. 624054 within the 7th European Community Framework Programme FP7/2007-2013 and by the European Research Council (ERC) under the European Union's Horizon 2020 research and innovation programme (Grants No. 341222 and No. 758759). D. M. is funded by the Spanish Grants No. FPA2014-52218-P and No. FPA2017-84436-P by Xunta de Galicia (GRC2013024), by FEDER and by the María de Maeztu Unit of Excellence MDM-2016-0692. D. A. is supported by the "Atracción del Talento" programme (Comunidad de Madrid) under Grant No. 2017-T1/TIC-5258 and by Severo Ochoa Programme Grants No. SEV-2016-0597 and No. FPA2015-65480-P (MINECO/FEDER). D. A. and D.M. thank the FRont Of pro-Galician Scientists for unconditional support.

*andrea.amoretti@ge.infn.it

†daniel.arean@uam.es

†blaise.gouteraux@polytechnique.edu

§aniele.musso@usc.es

[1] G. Grüner, The dynamics of charge-density waves, Rev. Mod. Phys. 60, 1129 (1988).

[2] B. I. Halperin and David R. Nelson, Theory of TwoDimensional Melting, Phys. Rev. Lett. 41, 121 (1978).

[3] L. V. Delacrétaz, B. Goutéraux, S. A. Hartnoll, and A. Karlsson, Theory of hydrodynamic transport in fluctuating electronic charge density wave states, Phys. Rev. B 96, 195128 (2017).
[4] B. Keimer, S. A. Kivelson, M. R. Norman, S. Uchida, and J. Zaanen, From quantum matter to high-temperature superconductivity in copper oxides, Nature (London) 518, 179 (2015).

[5] J. Zaanen and O. Gunnarsson, Charged magnetic domain lines and the magnetism of high- $T_{c}$ oxides, Phys. Rev. B 40, 7391 (1989).

[6] J. M. Tranquada, J. D. Axe, N. Ichikawa, Y. Nakamura, S. Uchida, and B. Nachumi, Neutron-scattering study of stripephase order of holes and spins in $\mathrm{la}_{1.48} \mathrm{nd}_{0.4} \mathrm{Sr}_{0.12} \mathrm{CuO}_{4}$, Phys. Rev. B 54, 7489 (1996).

[7] S. A. Kivelson, E. Fradkin, and V. J. Emery, Electronic liquid-crystal phases of a doped mott insulator, Nature (London) 393, 550 (1998).

[8] S. A. Kivelson, I. P. Bindloss, E. Fradkin, V. Oganesyan, J. M. Tranquada, A. Kapitulnik, and C. Howald, How to detect fluctuating stripes in the high-temperature superconductors, Rev. Mod. Phys. 75, 1201 (2003).

[9] M. Hücker, M. V. Zimmermann, G. D. Gu, Z. J. Xu, J. S. Wen, Guangyong Xu, H. J. Kang, A. Zheludev, and J. M. Tranquada, Stripe order in superconducting $\mathrm{la}_{2-x} \mathrm{ba}_{x} \mathrm{cuO}_{4}$ $(0.095 \leq x \leq 0.155)$, Phys. Rev. B 83, 104506 (2011).

[10] Y. Y. Peng, R. Fumagalli, Y. Ding, M. Minola, S. Caprara, D. Betto, M. Bluschke, G. M. De Luca, K. Kummer, E. Lefrançois, M. Salluzzo, H. Suzuki, M. Le Tacon, X. J. Zhou, N. B. Brookes, B. Keimer, L. Braicovich, M. Grilli, and G. Ghiringhelli, Re-entrant charge order in overdoped $(\mathrm{Bi}, \mathrm{Pb})_{2.12} \mathrm{Sr}_{1.88} \mathrm{CuO}_{6+\delta}$ outside the pseudogap regime, Nat. Mater. 17, 697 (2018).

[11] R. Arpaia, S. Caprara, R. Fumagalli, G. De Vecchi, Y. Y. Peng, E. Andersson, D. Betto, G. M. De Luca, N. B. Brookes, F. Lombardi, M. Salluzzo, L. Braicovich, C. Di Castro, M. Grilli, and G. Ghiringhelli, Dynamical charge density fluctuations pervading the phase diagram of a Cu-based high-Tc superconductor, Science 365, 906 (2019).

[12] E. W. Huang, C. B. Mendl, S. Liu, S. Johnston, H.-C. Jiang, B. Moritz, and T.P. Devereaux, Numerical evidence of fluctuating stripes in the normal state of high- $\mathrm{T}_{c}$ cuprate superconductors, Science 358, 1161 (2017).

[13] J. A. N. Bruin, H. Sakai, R. S. Perry, and A. P. Mackenzie, Similarity of scattering rates in metals showing t-linear resistivity, Science 339, 804 (2013).

[14] O. Gunnarsson, M. Calandra, and J. E. Han, Colloquium: Saturation of electrical resistivity, Rev. Mod. Phys. 75, 1085 (2003).

[15] S. Sachdev, Quantum Phase Transitions, 2nd ed. (Cambridge University Press, Cambridge, England, 2011).

[16] J. Zaanen, Why the temperature is high, Nature (London) 430, 512 (2004).

[17] N. E. Hussey, K. Takenaka, and H. Takagi, Universality of the Mott-Ioffe-Regel limit in metals, Philos. Mag. 84, 2847 (2004).

[18] L. Taillefer, Scattering and pairing in cuprate superconductors, Annu. Rev. Condens. Matter Phys. 1, 51 (2010).

[19] A. Abanov and A. V. Chubukov, Spin-Fermion Model Near the Quantum Critical Point: One-Loop Renormalization Group Results, Phys. Rev. Lett. 84, 5608 (2000).

[20] M. A. Metlitski and S. Sachdev, Quantum phase transitions of metals in two spatial dimensions. ii. spin density wave order, Phys. Rev. B 82, 075128 (2010). 
[21] D. F. Mross and T. Senthil, Theory of a Continuous Stripe Melting Transition in a Two-Dimensional Metal: A Possible Application to Cuprate Superconductors, Phys. Rev. Lett. 108, 267001 (2012).

[22] D. F. Mross and T. Senthil, Stripe melting and quantum criticality in correlated metals, Phys. Rev. B 86, 115138 (2012).

[23] L. V. Delacrétaz, B. Goutéraux, S. A. Hartnoll, and A. Karlsson, Bad metals from fluctuating density waves, SciPost Phys. 3, 025 (2017).

[24] J. M. Maldacena, The Large N limit of superconformal field theories and supergravity, Int. J. Theor. Phys. 38, 1113 (1999); Adv. Theor. Math. Phys. 2, 231 (1998).

[25] M. Ammon and J. Erdmenger, Gauge/Gravity Duality (Cambridge University Press, Cambridge, England, 2015).

[26] J. Zaanen, Y.-W. Sun, Y. Liu, and K. Schalm, Holo graphic Duality in Condensed Matter Physics (Cambridge University Press, Cambridge, England, 2015).

[27] S. A. Hartnoll, A. Lucas, and S. Sachdev, Holographic quantum matter, arXiv:1612.07324.

[28] H. Fukuyama and P. A. Lee, Pinning and conductivity of two-dimensional charge-density waves in magnetic fields, Phys. Rev. B 18, 6245 (1978).

[29] M. M. Fogler and D. A. Huse, Dynamical response of a pinned two-dimensional Wigner crystal, Phys. Rev. B 62, 7553 (2000).

[30] H. Fukuyama and P. A. Lee, Dynamics of the charge-density wave. i. impurity pinning in a single chain, Phys. Rev. B 17, 535 (1978).

[31] W. Finger and T. M. Rice, Long-wavelength phonons in incommensurate systems, Phys. Rev. B 28, 340 (1983).

[32] Y. Ling, C. Niu, J. Wu, Z. Xian, and H.-b. Zhang, MetalInsulator Transition by Holographic Charge Density Waves, Phys. Rev. Lett. 113, 091602 (2014).

[33] M. Baggioli and O. Pujolas, Electron-Phonon Interactions, Metal-Insulator Transitions, and Holographic Massive Gravity, Phys. Rev. Lett. 114, 251602 (2015).

[34] N. Jokela, M. Jarvinen, and M. Lippert, Pinning of holographic sliding stripes, Phys. Rev. D 96, 106017 (2017).

[35] T. Andrade, M. Baggioli, A. Krikun, and N. Poovuttikul, Pinning of longitudinal phonons in holographic spontaneous helices, J. High Energy Phys. 02 (2018) 085.

[36] L. Alberte, M. Ammon, M. Baggioli, A. Jiménez, and O. Pujolàs, Black hole elasticity and gapped transverse phonons in holography, J. High Energy Phys. 01 (2018) 129.

[37] T. Andrade, A. Krikun, K. Schalm, and J. Zaanen, Doping the holographic Mott insulator, Nat. Phys. 14, 1049 (2018).

[38] T. Andrade and A. Krikun, Coherent vs incoherent transport in holographic strange insulators, J. High Energy Phys. 05 (2019) 119.

[39] P. K. Kovtun, D. T. Son, and A. O. Starinets, Viscosity in Strongly Interacting Quantum Field Theories from Black Hole Physics, Phys. Rev. Lett. 94, 111601 (2005).

[40] R. A. Davison, K. Schalm, and J. Zaanen, Holographic duality and the resistivity of strange metals, Phys. Rev. B 89, 245116 (2014).

[41] J. Zaanen, Planckian dissipation, minimal viscosity and the transport in cuprate strange metals, SciPost Phys. 6, 061 (2019).
[42] A. Donos and J.P. Gauntlett, Holographic Q-lattices, J. High Energy Phys. 04 (2014) 040.

[43] A. Amoretti, D. Areán, R. Argurio, D. Musso, and L. A. Pando Zayas, A holographic perspective on phonons and pseudo-phonons, J. High Energy Phys. 05 (2017) 051.

[44] A. Amoretti, D. Areán, B. Goutéraux, and D. Musso, Effective holographic theory of charge density waves, Phys. Rev. D 97, 086017 (2018).

[45] A. Amoretti, D. Areán, B. Goutéraux, and D. Musso, DC Resistivity of Quantum Critical, Charge Density Wave States from Gauge-Gravity Duality, Phys. Rev. Lett. 120, 171603 (2018).

[46] A similar model was considered in [47] to study spin density waves. Previous recent holographic studies of collective transport in phases that break translations spontaneously include [34-37,48,49].

[47] N. Iqbal, H. Liu, M. Mezei, and Q. Si, Quantum phase transitions in holographic models of magnetism and superconductors, Phys. Rev. D 82, 045002 (2010).

[48] L. Alberte, M. Ammon, A. Jiménez-Alba, M. Baggioli, and O. Pujolàs, Holographic Phonons, Phys. Rev. Lett. 120, 171602 (2018).

[49] N. Jokela, M. Jarvinen, and M. Lippert, Holographic sliding stripes, Phys. Rev. D 95, 086006 (2017).

[50] See Supplemental Material at http://link.aps.org/ supplemental/10.1103/PhysRevLett.123.211602, which includes Refs. [1,3,23,28-31,40,44,45,48,51-63] and where we give a brief review of Wigner crystal hydrodynamics, details of our numerical analysis and technical derivations of some of our results in the main text.

[51] P. M. Chaikin and T. C. Lubensky, Principles of Condensed Matter Physics (Cambridge University Press, Cambridge, England, 1995).

[52] A. Amoretti, D. Areán, B. Goutéraux, and D. Musso, Diffusion and universal relaxation of holographic phonons, J. High Energy Phys. 10 (2019) 068.

[53] B. Goutéraux, Charge transport in holography with momentum dissipation, J. High Energy Phys. 04 (2014) 181.

[54] R. J. Anantua, S. A. Hartnoll, V. L. Martin, and D. M. Ramirez, The Pauli exclusion principle at strong coupling: Holographic matter and momentum space, J. High Energy Phys. 03 (2013) 104.

[55] A. J. Beekman, J. Nissinen, K. Wu, K. Liu, R.-J. Slager, Z. Nussinov, V. Cvetkovic, and J. Zaanen, Dual gauge field theory of quantum liquid crystals in two dimensions, Phys. Rep. 683, 1 (2017).

[56] L. P. Kadanoff and P. C. Martin, Hydrodynamic equations and correlation functions, Ann. Phys. (N.Y.) 24, 419 (1963).

[57] P. Kovtun, Lectures on hydrodynamic fluctuations in relativistic theories, J. Phys. A 45, 473001 (2012).

[58] M. Kaminski, K. Landsteiner, J. Mas, J. P. Shock, and J. Tarrio, Holographic operator mixing and quasinormal modes on the brane, J. High Energy Phys. 02 (2010) 021.

[59] A. Donos, D. Martin, C. Pantelidou, and V. Ziogas, Hydrodynamics of broken global symmetries in the bulk, arXiv: 1905.00398.

[60] G. Policastro, D. T. Son, and A. O. Starinets, From AdS/CFT correspondence to hydrodynamics, J. High Energy Phys. 09 (2002) 043. 
[61] G. Policastro, D. T. Son, and A. O. Starinets, From AdS/CFT correspondence to hydrodynamics. 2. Sound waves, J. High Energy Phys. 12 (2002) 054.

[62] C. P. Herzog, The sound of M theory, Phys. Rev. D 68, 024013 (2003).

[63] D. T. Son and A. O. Starinets, Minkowski space correlators in AdS/CFT correspondence: Recipe and applications, J. High Energy Phys. 09 (2002) 042.

[64] A. Donos, J.P. Gauntlett, T. Griffin, and V. Ziogas, Incoherent transport for phases that spontaneously break translations, J. High Energy Phys. 04 (2018) 053.

[65] Similar Gell Mann-Oakes-Renner relations have previously been obtained in holography and in field theory $[38,43,66]$.

[66] D. Musso, Simplest phonons and pseudo-phonons in field theory, arXiv:1810.01799.

[67] A. Donos and J. P. Gauntlett, Novel metals and insulators from holography, J. High Energy Phys. 06 (2014) 007.

[68] R. A. Davison and B. Goutéraux, Dissecting holographic conductivities, J. High Energy Phys. 09 (2015) 090.

[69] R. A. Davison and B. Goutéraux, Momentum dissipation and effective theories of coherent and incoherent transport, J. High Energy Phys. 01 (2015) 039.

[70] R. A. Davison, B. Goutéraux, and S. A. Hartnoll, Incoherent transport in clean quantum critical metals, J. High Energy Phys. 10 (2015) 112.

[71] R. A. Davison, S. A. Gentle, and B. Goutéraux, Slow Relaxation and Diffusion in Holographic Quantum Critical Phases, Phys. Rev. Lett. 123, 141601 (2019).

[72] R. A. Davison, S. A. Gentle, and B. Goutéraux, Impact of irrelevant deformations on thermodynamics and transport in holographic quantum critical states, Phys. Rev. D 100, 086020 (2019).

[73] K. Wang, N. Bachar, J. Teyssier, W. Luo, C. W. Rischau, G. Scheerer, A. de la Torre, R. S. Perry, F. Baumberger, and
D. van der Marel, Mott transition and collective charge pinning in electron doped $\mathrm{Sr}_{2} \mathrm{IrO}_{4}$, Phys. Rev. B 98, 045107 (2018).

[74] A. Charnukha, D. Pröpper, N. D. Zhigadlo, M. Naito, M. Schmidt, Z. Wang, J. Deisenhofer, A. Loidl, B. Keimer, A. V. Boris, and D. N. Basov, Intrinsic Charge Dynamics in High- $T_{c} a \mathrm{FeAs}(\mathrm{O}, \mathrm{F})$ Superconductors, Phys. Rev. Lett. 120, 087001 (2018).

[75] S. A. Hartnoll, Theory of universal incoherent metallic transport, Nat. Phys. 11, 54 (2015).

[76] J. Zhang, E. M. Levenson-Falk, B. J. Ramshaw, D. A. Bonn, R. Liang, W. N. Hardy, S. A. Hartnoll, and A. Kapitulnik, Anomalous thermal diffusivity in underdoped $\mathrm{YBa}_{2} \mathrm{Cu}_{3} \mathrm{O}_{6+x}$, Proc. Natl. Acad. Sci. U.S.A. 114, 5378 (2017).

[77] J. Zhang, E. D. Kountz, E. M. Levenson-Falk, R. L. Greene, and A. Kapitulnik, Thermal diffusivity above Mott-IoffeRegel limit, arXiv:1808.07564.

[78] P. T. Brown, D. Mitra, E. Guardado-Sanchez, R. Nourafkan, A. Reymbaut, S. Bergeron, A. M. S. Tremblay, J. Kokalj, D. A. Huse, P. Schauss, and W. S. Bakr, Bad metallic transport in a cold atom Fermi-Hubbard system, Science 363, 379 (2019).

[79] L. V. Delacrétaz, B. Goutéraux, S. A. Hartnoll, and A. Karlsson, Theory of the collective magnetophonon resonance and melting of the field-induced Wigner solid, Phys. Rev. B 100, 085140 (2019).

[80] A. Lucas, Hydrodynamic transport in strongly coupled disordered quantum field theories, New J. Phys. 17, 113007 (2015).

[81] A. Donos and C. Pantelidou, Holographic transport and density waves, J. High Energy Phys. 05 (2019) 079. 